\title{
Sensitivity Mapping for Electrical Tomography Using Finite Element Method
}

\author{
Hanis Liyana Mohmad Ameran', Ahmad Azahari Hamzah², Ruzairi Abdul \\ Rahim ${ }^{1}$, Fatin Aliah Phang ${ }^{3}$, Nor Hasrul Akhmal Bin Ngadiman ${ }^{4}$, Yasmin \\ Abdul Wahab ${ }^{5}$, Jaysuman Pusppanathan ${ }^{6 *}$
}

\author{
${ }^{1}$ Faculty of Electrical and Electronic Engineering, Universiti Tun Hussein Onn Malaysia, 86400 Batu Pahat, Johor, \\ Malaysia \\ ${ }^{2}$ Section of chemical emgineering technology, UniKL Micet, 78000 Alor Gajah Melaka \\ ${ }^{3}$ Centre of Engineering Education (CEE), Universiti Teknologi Malaysia, 81300 Skudai Johor, Malaysia. \\ ${ }^{4}$ Faculty of Mechanical Engineering, Universiti Teknologi Malaysia. 81310 Skudai Johor, Malaysia \\ ${ }^{5}$ Instrumentation and Control Engineering department (ICE), Faculty of Electrical and Electronics Engineering \\ Universiti Malaysia Pahang, 26600 Pekan, Pahang, Malaysia \\ ${ }^{6}$ Faculty of Biosciences and Medical Engineering, Universiti Teknologi Malaysia, 81310 Skudai Johor, Malaysia
}

Received 21 January 2018; accepted 10 June 2018, available online 6 August 2018

\begin{abstract}
This paper presents the construction of sensitivity map (SM) using finite element method for electrical tomography systems that has the soft-field characteristic such as electrical capacitance tomography (ECT). The SM is generated based on a sixteen-channel ECT system on a $110 \mathrm{~mm}$ diameter pipe circumference by using COMSOL Multiphysic. Sensitivity mapping is the pivotal element for tomography imaging in solving the forward problem. The obtained sensitivity map for 120 projections forming a sensitivity matrices are presented in this paper.
\end{abstract}

Keywords: Sensitivity map, tomography, electrical capacitance

\section{Introduction}

Electrical Capacitance Tomography (ECT) is a technique for cross-sectional visualization of the distributed dielectric materials within the sensing region. It is based on measuring the changes of inter-electrode capacitance of the distributed field. Commonly, is it applied in a multiphase flow formed by elements of different permittivity [1]. From the measured data, a reconstructed image of the permittivity distribution inside the vessel can be obtained via an image reconstruction algorithm such as linear back projection or tikhonov regularization method. A typical tomography system can be describe as in figure $1[2]$.

\section{The basic principle of ECT}

The basic principle of ECT system is to detect the capacitance changes within an electric potential distributed field. A simple and most effective applications of such system is to measure the capacitances changes on a parallel plate electrode configuration [3] as in figure.1.

The basic buildup of an ECT system will consist of a sensory system, signal processing circuitry and software interfacing where image reconstruction work take place.
The process flow of ECT is depicted in figure 2 follows [2, $5]$.

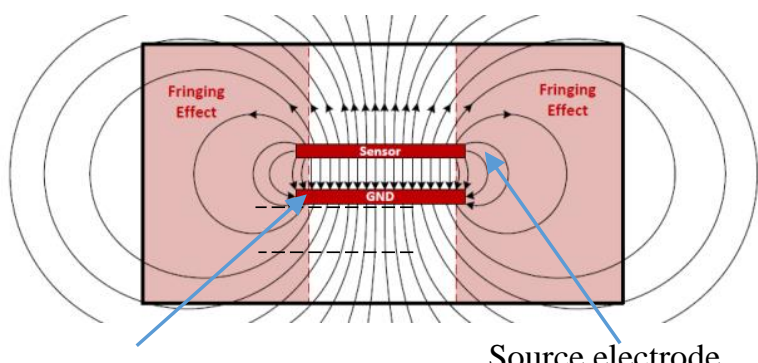

Detecting electrode

Source electrode

Fig. 1. Electric fields of a parallel plate electrodes

The energized capacitive plate will have a uniform electric field between the electrodes far from their edges but these field lines form fringes at the edge of these plates known as fringe effect which can be removed by encircling the sensing electrodes with a guard electrode [4]. By applying Gauss' law, the charge measurement of the inter-electrode capacitances can be determined from equation (1). Further understanding of ECT modelling is explained in [5]. 


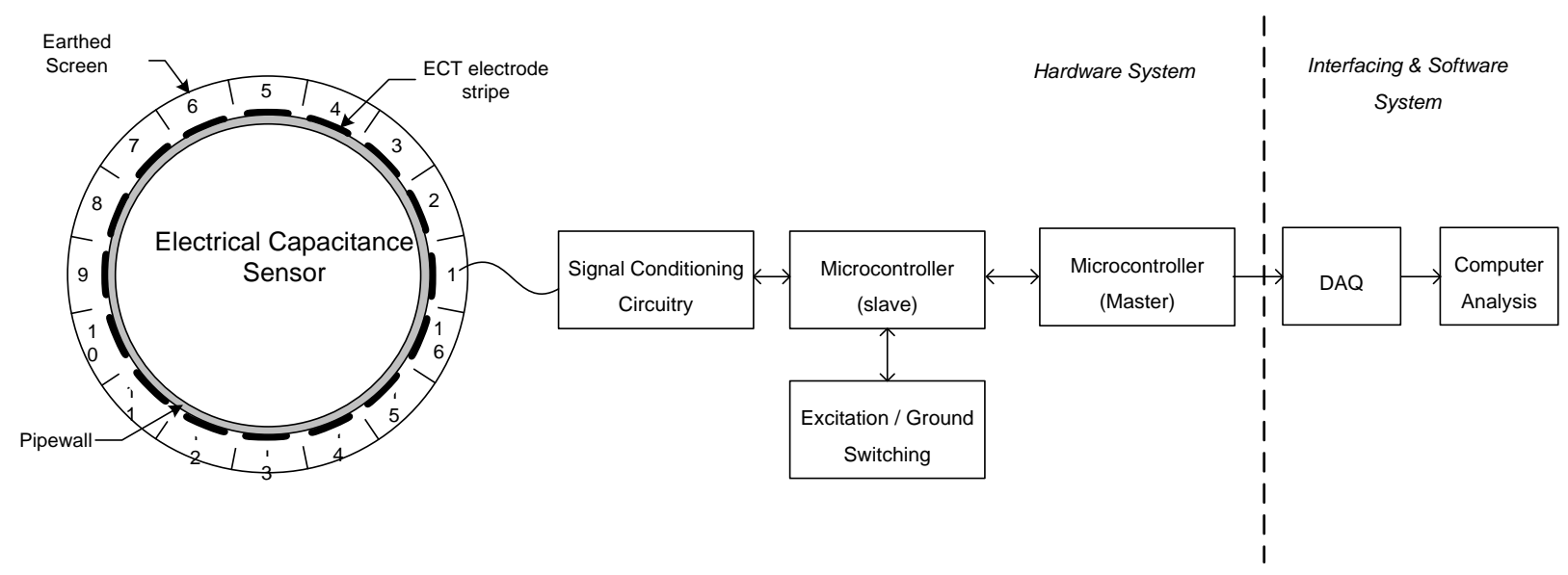

Fig. 2. Electrical capacitance tomography system

$$
C=\frac{\varepsilon_{0} \varepsilon_{r} A}{d_{p}}
$$

Where $\varepsilon_{0}$ is the permittivity of vacuum space, $\varepsilon_{\mathrm{r}}$ is the permittivity of the dielectric material between the measurement electrodes, $d_{\mathrm{p}}$ is the distance between the electrodes, $A$ is the area of the sensing plate.

Referring to figure 3, unlike hard-field system, the softfield system has electric potential field line propagates from a source electrode (e1) in a curved line and further curves away between the two different dielectric materials (liquid $\varepsilon_{2}$ and air $\varepsilon_{2}$ ).

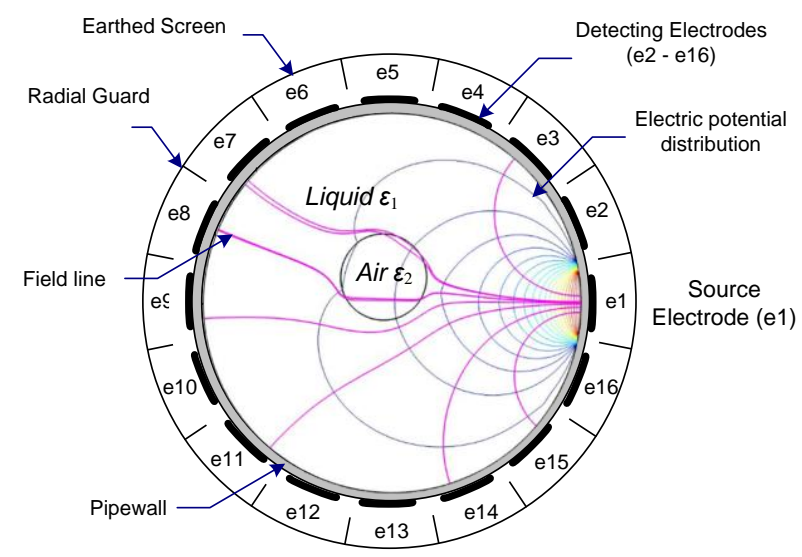

Fig. 3: Soft-field effect with two different dielectric materials

The presents of different dielectric materials in this inhomogeneous condition changes the space distribution thus causes capacitance changes between the electrodes. The capacitance between electrodes are measured between each source and detector electrode sequentially with a sum of 120 independent measurements obtained with equation (2) [6-8].

$$
N=\frac{n(n-1)}{2}
$$

where $\mathrm{N}$ is the total number of electrodes. The increasing number of electrodes generally could improve the resolution of image result but in practice, higher number of sensor will trade-off in decreasing the sensing size of the sensor to fit on a pipe circumference thus reduces the sensitivity to capture the inter-electrode capacitance.

\section{Forward Problem}

The forward problem is addressed to determine the theoretical sensor data by discretizing an image plane into a grid of $128 \times 128$ pixels for each sensor projections (e1 to e16) [79].

Therefore, the $100 \mathrm{~mm}$ diameter pipe is discretized into an image plane which consist of 16384 element array of which 13076 effective pixels within the boundary circle or within the region of interest while 3308 pixels are ineffective as in figure 4.

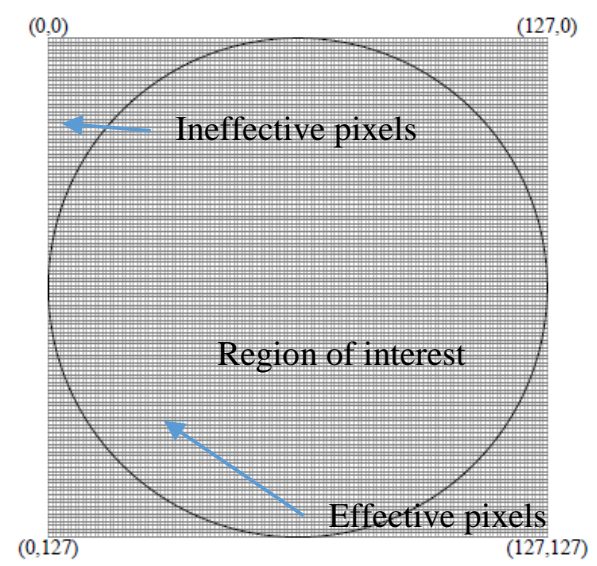

Fig. 4. 2D discretized image plane of $128 \times 128$ pixels 
The discretized image plane in pixels will be used to display the sensitivity distribution of the normalized composite permittivity (ECT) of each pixel to construct images. Therefore, the forward problem can be solved by modelling the ECT sensor projections to its corresponding sensitivity map matrices $[9,10]$.

\section{FEM Modelling}

The basis of the finite element method was developed as a way of decomposing complex problems into simple well known parts. COMSOL Multiphysics software is able to carry out FEM to solve physical systems of immense complexity through approximated simulation of the system geometry. The FEM setup includes following steps to generate the sensitivity maps for 120 projections [11].

$$
\begin{array}{ll}
\text { - } & \text { Sensor geometry modelling } \\
\text { - } & \text { Material selection and assignment } \\
\text { Pre-processing work }
\end{array}
$$

The electrostatic multiphysics (ES) is selected for the whole FEM analysis. By using COMSOL, an ECT geometry model is created to virtualize the sensor projections in two dimensional view with its actual size and dimension as in figure 5 .

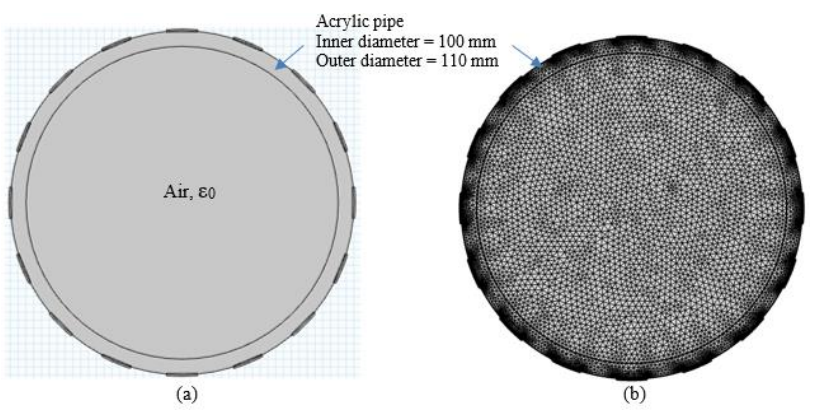

Fig. 5. 16-channel ECT configuration (a) Geometry model (b) Meshed geometry

To obtain a smooth projection map, extra fine meshing with maximum element size of $0.222 \mathrm{~mm}$ and minimum $44 \mathrm{e}^{-3} \mathrm{~mm}$ under meshing physics controlled setting is chosen since denser meshing would provide better image reconstruction. The relationship between the capacitance and the permittivity distribution is governed by Poisson equation as follows [12].

$$
\nabla \cdot[\varepsilon(r) \nabla \varphi(r)]=0
$$

Where $\varepsilon(r)=$ spatial permittivity distribution and $\varphi(r)$ $=$ potential distribution. The boundary conditions imposed on the model has an electric potential $\varphi(r)=\Delta V_{i j}$ where $\Delta V_{i j}$ is the potential difference between the excitation electrode $i$ and detection electrode $j$. Meanwhile, the remaining electrodes are virtually set as ground where $\varphi(r)=0$. The electrical charge $Q_{i j}$ on the detecting electrodes can be calculated using Gauss surface integral law as in equation (4) thus the inter-electrode capacitance is as equation (5) [14].

$$
\begin{aligned}
& Q_{i j}=\oint_{S} \varepsilon(r) \cdot \nabla \varphi(r) d s \\
& \mathrm{C}_{i j}=\frac{1}{\Delta \mathrm{V}_{i j}} \oint_{S} \varepsilon(r) \cdot \nabla \varphi(r) d s
\end{aligned}
$$

where $s=$ electrode surface. Electrostatic interface model will simulate and solve these complex numerical problems. This process will be repeated for each electrode until all 120 projections are measured. As a result, the electric field distribution of the projection beam from one sensor to another will be intensified as in figure 6. It shows the generated sensitivity map for the permittivity distribution for electrode e1 to e5.

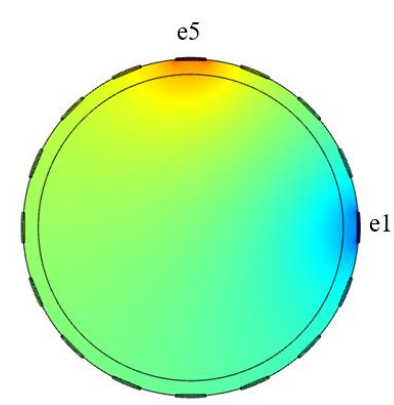

(a)

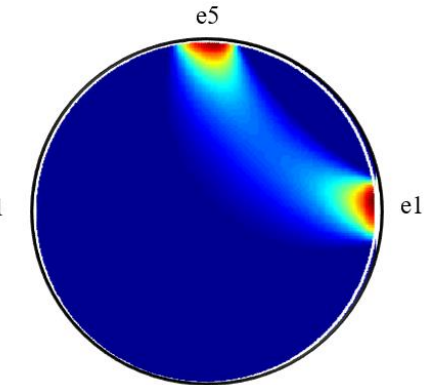

(b)
Fig. 6. Sensitivity map projection e1 to e5 (a) projection in COMSOL (b) refined projection using Matlab

\section{Results and Discussion}

The other projections from an excitation electrode $e_{i}$ to the detectors $e_{j}$ are depicted in figure 7 follows. Since the circular electrode positions are symmetrical, only 120 independent projections are required.

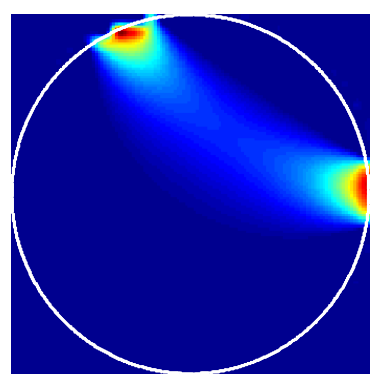

(a) e1 to e7

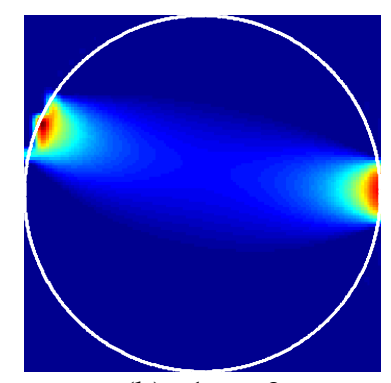

(b) e1 to e9 


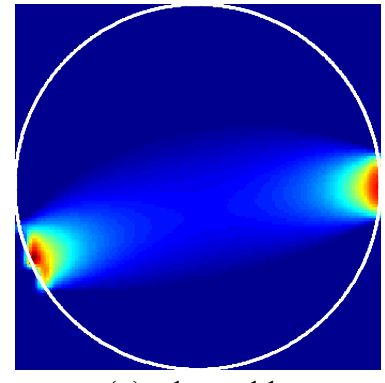

(c) e1 to e11

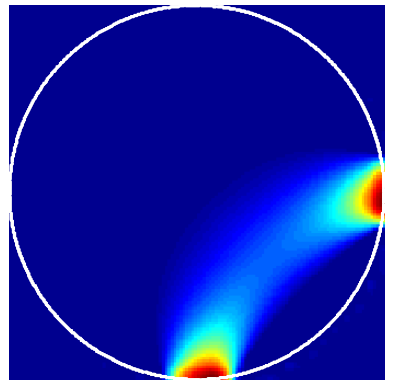

(d) e1 to e13

Fig.7. Sensitivity distribution projections for ECT

From figure 7 , it is observed that the sensitivity field is non-uniformly distributed over the medium of interest. The sensitivity is higher within the area closer to the excitation and detection electrode pair. On the other hand, its sensitivity is much lower in the center region. Therefore, the drawback of this soft-field effect will affect the measurements on the center region.

\section{Conclusion}

This paper presented a method to generate sensitivity distribution of an electrical capacitance tomography system using COMSOL multiphysic software; a finite element analysis software. The complex forward problem can be solved using COMSOL by visualizing the crosssectional image on a discretized image plane of $128 \times 128$ pixel for high spatial resolution image. All 120 sensitivity maps formed up a sensitivity matrices which will be used for image reconstruction work. The generated sensitivity map will be useful for image reconstruction task by using a specific algorithm such as linear back projection.

\section{Acknowledgement}

The authors would like to acknowledge the Research University grant (GUP) 02K97, 16H50, 03G77, 4B311, $02 \mathrm{~K} 87$ for funding and supporting this research. Authors are also grateful to process tomography research group (PROTOM) UTM.

\section{References}

[1] Zhiyao Huang, B.W.a.H.L., Application of electrical capacitance tomography to the void fraction measurement of two-phase flow. IEEE Transactions on Instrumentation and Measurement,, 2003. 52(1): p. 7-12.

[2] Pusppanathan, J., et al., Finite Element Analysis on Electrical Capacitance Sensor Guard. Man-Machine Systems Vol. 2, 2015. 77(28): p. 61-65.

[3] Baxter, L.K., Capacitive Sensors Design and Application, ed. R.J. Herrick. 1997: IEEE Press.

[4] Hegg, M.C. and A.V. Mamishev, Influence of Variable Plate Separation on Fringing Electric Fields in ParallelPlate Capacitors, in International Symposium on Electrical Insulation. 2004: Indianapolis, USA. p. 19-22.

[5] Pusppanathan, J., et al., Single-Plane Dual Modality Tomography for Multiphase Flow Imaging by Integrating Electrical Capacitance and Ultrasonic Sensors. IEEE Sensors Journal, 2017. 17(19): p. 6368 - 6377.

[6] Shafquet, A., I. Ismail, and A. Jaafar, Modeling and simulation of multi-plane Electrical Capacitance Tomography sensor for flow imaging by using Finite Element Analysis, in 5th International Conference on Intelligent and Advanced Systems (ICIAS). 2014, IEEE: Kuala Lumpur, Malaysia. p. 1-6.

[7] Taylor, S.H. and S.V. Garimella, Design of electrode arrays for $3 D$ capacitance tomography in a planar domain. International Journal of Heat and Mass Transfer, 2017. 106: p. 1251-1260.

[8] Seong, C.K., et al., Hardware Development of Electrical Capacitance Tomography (ECT) System with Capacitance Sensor for Liquid Measurements. Jurnal Teknologi, 2015. 73(6).

[9] Rahiman, M.H.F., et al., A Study on Forward and Inverse Problems for an Ultrasonic Tomography. Special Issue on Advanced Sensors and Instrumentation Systems, 2014. 70(3).

[10] Yong Song, K. and et al., Sensitivity map generation in electrical capacitance tomography using mixed normalization models. Measurement Science and Technology, 2007. 18(7): p. 2092.

[11] Pusppanathan, J., et al., 3D Modelling of Electrical Capacitance and Ultrasonic Sensor Integration Using Finite Element Method. Special Issue on Sensor Technology and Control System Applications Vol. 3, 2015. 77(17): p. 31-35.

[12] Ismail, I., et al., Tomography for multi-phase flow measurement in the oil industry. Flow Measurement and Instrumentation, 2005(16): p. 145-155. 